\title{
LIPOSARCOMA RETROPERITONEAL CALCIFICADO
}

\author{
R. FERRERO DORIA, F. MORENO PÉREZ, E. HUERTAS VALERO, F. GARCÍA VÍCTOR, \\ M. GASSÓ MATOSES, S. CALATRAVA GADEA, E. DÍAZ CALLEJA
}

Servicio de Urología. Hospital Francesc de Borja de Gandia. Valencia.

Actas Urol Esp. 28 (3): 234-237, 2004

\section{RESUMEN \\ LIPOSARCOMA RETROPERITONEAL CALCIFICADO}

Se presenta un caso acontecido en nuestro centro correspondiente a un liposarcoma retroperitoneal pleomórfico calcificado en forma de cáscara. Se describe la forma de presentación, los síntomas por los que consulta el paciente, los métodos diagnósticos utilizados y la alternativa terapéutica tomada ante este caso. Se discuten los posibles diagnósticos diferenciales a los que obliga este caso clínico.

PALABRAS CLAVE: Liposarcoma retroperitoneal. Tumores retroperitoneales. Sarcomas. Calcificación.

\section{ABSTRACT}

RETROPERITONEAL LIPOSARCOMA UIT CALCIFICATION

Case report of a retroperitoneal liposarcoma pleomorfico with calcification in diagnosed in our hospital. We comment on its clinical characteristics, diagnostic methods and treatment. We likewise comment the possible differential diagnoses.

KEY WORDS: Retroperitoneal liposarcoma. Retroperitoneal tumors. Sarcomas. Calcification.

$\mathrm{L}$ a presencia de lesiones calcificadas en el retroperitoneo obliga a pensar en una lesión de origen renal, sin poder descartar cualquier otro origen. Existen múltiples publicaciones que describen casos de lesiones retroperitoneales con calcificaciones en su interior en forma estrellada, en cáscara de huevo,... que están presentes en el interior de la masa retroperitoneal. Una de las posibilidades es la presencia de un sarcoma que albergue en su interior calcificaciones, como ya han sido descritas en otras publicaciones.

Los sarcomas son una variedad de tumores cuyo origen es el mesénquima y que constituye entre $7-25 \%$ de los tumores malignos del retroperitoneo. Han sido descritos casos de sarcomas retroperitoneales primitivos de este espacio así como otros cuyo origen es el mismo riñón.
El liposarcoma es un tumor maligno derivado del tejido graso, de frecuente localización retroperitoneal y que obliga al diagnóstico diferencial con el angiomiolipoma o con lipoma benigno primario.

Suelen manifestarse clínicamente por sintomas constitucionales y por la compresión que ejercen sobre estructuras vecinas, más frecuentemente digestivas.

En este artículo se presenta un caso acontecido en nuestro centro cuyo interés viene centrado en la espectacularidad de la calcificación del liposarcoma retroperitoneal.

\section{CASO CLÍNICO}

Varón de 69 años que acude a nosotros para revisión de síndrome prostático en tratamiento con un alfa-bloqueante. Como antecedentes destaca 
haber sido intervenido de cadera derecha, hipoacusia y angor repetido. Episodio de traumatismo costolumbar izquierdo hacía unos 30 años. Como antecedentes urológicos hematuria en dos ocasiones, la última vez hacía un año.

En la exploración la próstata es de características fibroadenomatosas, volumen II/IV, sin nodulaciones. Destaca a la palpación abdominal gran masa situada en hipocondrio y vacío izquierdo, dolorosa a la palpación.

Analítica: Hb 14,6 gr/dl, Hcto. 43,1\%, VSG 9 $\mathrm{mm} / \mathrm{h}$ y bioquímica dentro de la normalidad. PSA: $3,1 \mathrm{ng} / \mathrm{ml}$.

Rx simple: gran efecto masa situada en hipocondrio y vacío izquierdo, de forma ovoide totalmente calcificada (Fig. 1).

Ecografia abdominal: gran masa suprarrenal izquierda, riñones y vejiga normales, próstata de $5,4 \times 6,6 \times 6,8$ (133 cc).

TAC abdominal: masa suprarrenal izquierda de $16 \times 16 \times 14 \mathrm{~cm}$ de diámetro aproximadamente, que desplaza riñón izquierdo, bazo, colon descendente y páncreas, con extensa corona de calcificación grosera anular de un grosor aproximado de entre 3-4 cm y adoptando patrón estriado (Fig. 2).

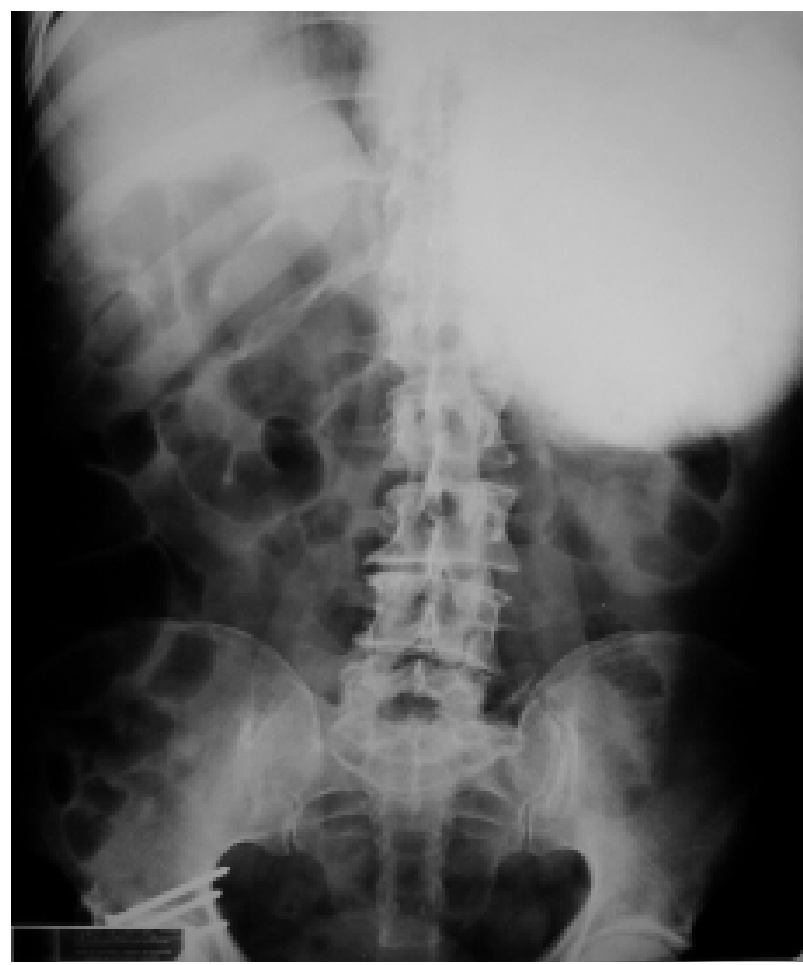

FIGURA 1. Rx simple de aparato urinario con imagen de masa calcificada en hipocondrio y vacio izquierdo.

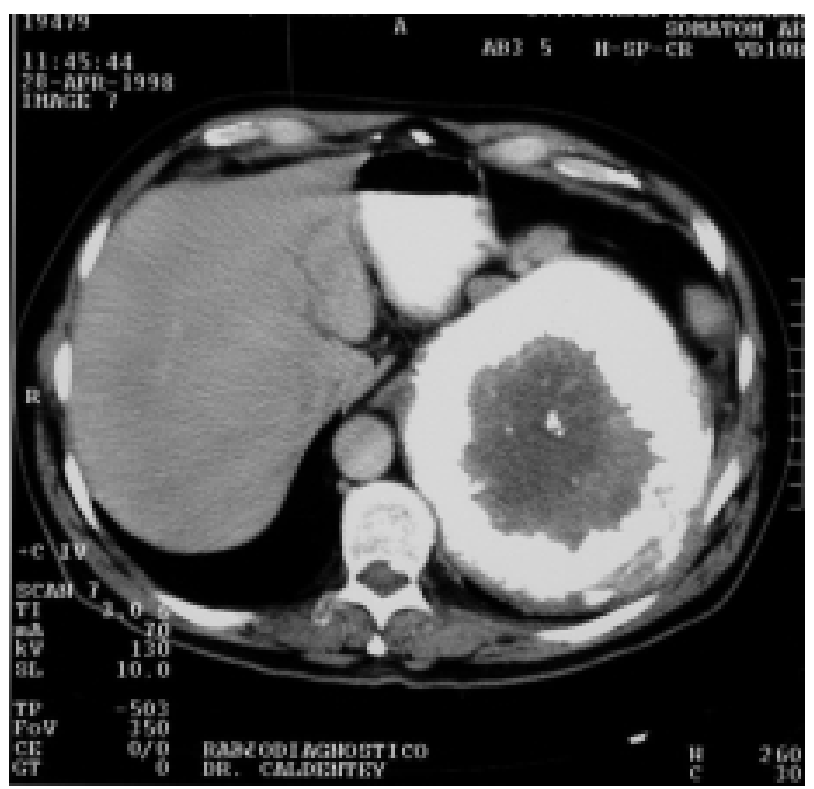

FIGURA 2. TAC abdominal: masa retroperitoneal con calcificación grosera en corona y contenido hipodenso.

Ante estos hallazgos se plantea diagnóstico diferencial con cualquier masa situada en el retroperitoneo que presente este tipo de calcificación:

- Tumores retroperitoneales primitivos

- Tumores suprarrenales

- Tumores renales

- Tumores metastásicos

- Patología infecciosa

Se realizan determinaciones sanguíneas de actividad de renina plasmática antes y después de la deambulación, así como la aldosterona y cortisol a las 8 y 20 horas, siendo los resultados dentro de la normalidad. Los niveles en orina de 17-cetosteroides y corticosteroides, así como las catecolaminas, metanefrinas y vanilmandelato eran normales. Todo lo anterior descartaba tumor adrenal funcionante.

También se realizaron tinciones para bacilos ácido alcohol resistentes en orina y esputo siendo negativo, así como el test de Mantoux.

Se planteó la posibilidad de quiste hidatídico calcificado, pero la serología era negativa.

Asímismo se pensó en el traumatismo costolumbar izquierdo, antes sufrido, como posible causa de la masa suprarrenal calcificada.

Con el diagnóstico de masa retroperitoneal sin filiar se practicó laparotomía exploradora encontrando lesión sobresaliente en hipocon- 
drio izquierdo, dura a la palpación, con fijación a planos profundos y lesiones difusas repartidas por todo el peritoneo, siendo el informe anatomopatológico intraoperatorio de las mismas de grasa peritoneal infiltrada por tumor maligno de probable origen mesenquimal. Se decide cierre de cavidad abdominal al tratarse de un proceso maligno diseminado. El informe patológico definitivo es de liposarcoma pleomórfico, siendo las técnicas inmunohistoquímicas de: S100: positiva; Oil-Red: positiva; vimentina: positiva; PS 3: positiva; desmina: negativa (Fig. 3).

El paciente es dado de alta al séptimo día de la intervención, falleciendo posteriormente a las dos semanas en su domicilio.

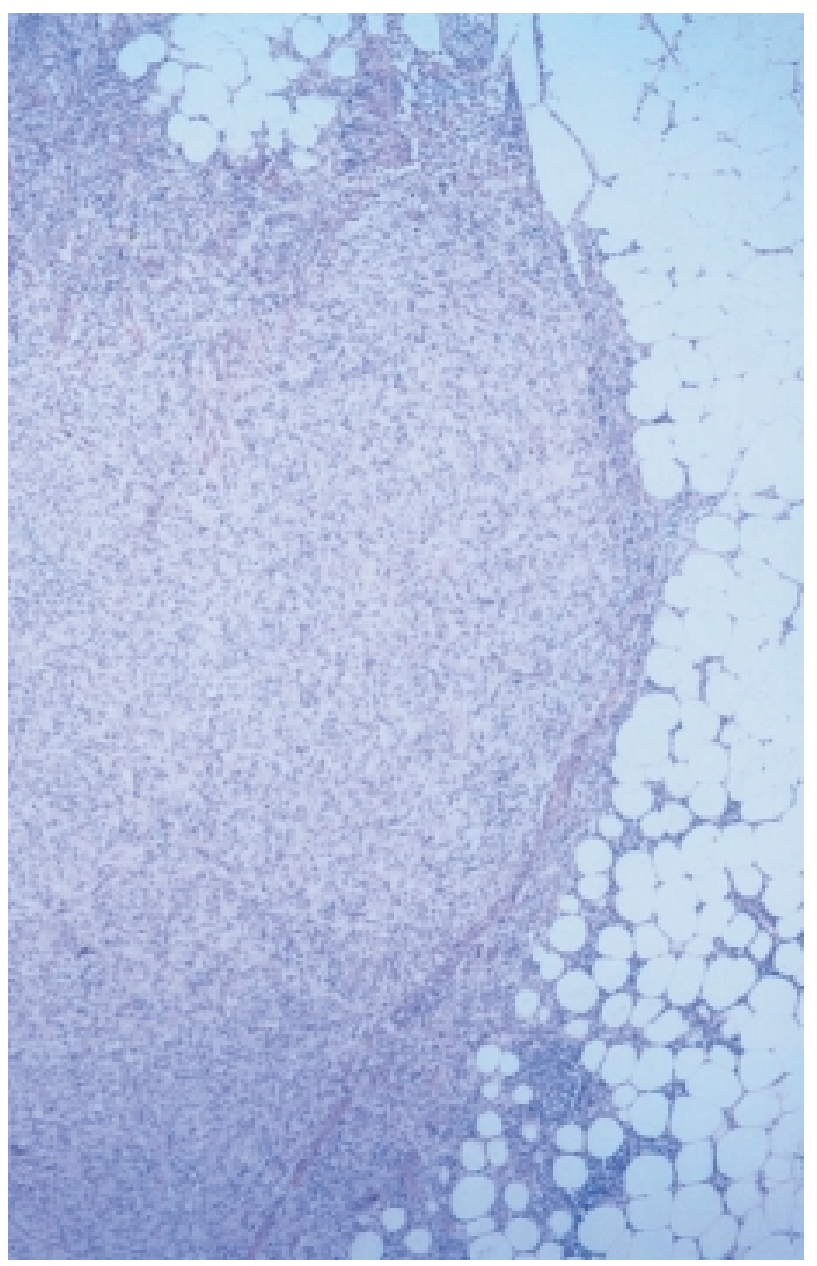

FIGURA 3. Preparación patológica: a la izquierda proliferación sarcomatosa infiltrando el tejido graso circundante; a la derecha los elementos tumorales son de aspecto "lipoblástico" con actividad mitótica incrementada.

\section{DISCUSIÓN Y COMENTARIOS}

Los tumores retroperitoneales primitivos son unas neoplasias de etiología desconocida, poco frecuentes en el organismo suponiendo entre 0,07-0,2\% de los tumores del mismo ${ }^{1,2}$.

El liposarcoma es un tumor que tiene su origen en células adiposas del primitivo tejido mesodérmico, poco frecuente, constituyendo alrededor el $7-25 \%$ de los tumores del retroperitoneo y que aparece con mayor frecuencia en varones entre la cuarta y sexta década de la vida ${ }^{3}$.

Podemos distinguir cuatro variedades anatomopatológicas: bien diferenciado, mixoide, lipoblástico o de células redondas y pleomórfico, siendo esta última variedad la de peor pronóstico $^{4}$.

Es habitual ser diagnosticadas cuando han adquirido grandes dimensiones. Las manifestaciones clínicas a que da lugar suelen derivarse del espacio que ocupan durante su crecimiento: manifestaciones eminentemente digestivas (dolor abdominal, náuseas, vómitos), como masa palpable a la exploración en un alto porcentaje $(78 \%)^{5}$, fiebre, pérdida de peso $^{6}, \ldots$ Puede manifestarse en forma de sintomas paraneoplásicos como ginecomastia o varicocele ${ }^{5}$. Cabe destacar en nuestro caso que a pesar del tamaño de la masa el paciente no refirió en ningún momento estos síntomas exceptuando el de dolor abdominal a la palpación.

Desde el punto de vista diagnóstico suele ser la ecografía la que nos pone en la pista para posteriormente realizar un TAC o RMN para el estudio de esta masa, teniendo como objetivo conocer su extensión, topografía y relación con órganos vecinos, características de la $\operatorname{masa}^{5}, \ldots$ siendo el liposarcoma una tumoración con patrones radiológicos bastante definitivos tras el estudio con el TAC, debido a que el tejido adiposo aparece como hipodenso. En el caso que nos ocupa ofrecía un grado mayor de dificultad debido a la enorme cáscara cálcica que rodeaba el mismo y que contrariamente a lo que expresan otros autores, la calcificación no fue signo de buen pronóstico ${ }^{7}$.

Ante masas retroperitoneales de estas características, que en nuestro caso inicialmente son informadas de masa suprarrenal, estamos obligados a descartar cualquier carcinoma funcionante o no de la glándula suprarrenal, aunque es 
bien sabido que en casi todas estas masas retroperitoneales el diagnóstico de certeza se obtiene tras el análisis patológico de la pieza quirúrgica.

En este caso, debido a la importante calcificación que rodeaba el tumor, teníamos que descartar así mismo todas aquellas entidades de posible localización retroperitoneal calcificadas: hemangiopericitomas, tumores neuroblásticos, enfermedad de Castleman, adenoma adrenal hemorrágico, linfangionas, teratomas, quistes hidatídicos, incluso lesión benigna de glándula suprarrenal calcificada después de un traumatismo.

El tratamiento es quirúrgico con la intención de practicar una cirugía lo más radical posible conociendo el alto porcentaje de recidivas locales y asumiendo la necesidad de extirpar órganos vecinos de forma parcial o total. Así suele ser necesario realizar nefrectomía en un $25 \%$ de los casos y colectomía en un $20 \% 6$.

La aplicación de radioterapia inicial como forma de tratamiento en los sarcomas retroperitoneales rara vez es efectiva dado que los tumores mesodérmicos son radiorresistentes ${ }^{8}$.

En cuanto al pronóstico de los tumores retroperitoneales malignos la supervivencia oscila alrededor del $40 \%$ tras una resección completa y solamente del $5 \%$ cuando no puede resecarse todo el tumor, lo cual ocurre en un porcentaje de alrededor del 25-65\% de los casos ${ }^{5}$.

\section{REFERENCIAS}

1. CALO PG, CONGUI A, FERRELI C et al.: I tumori retroperitoneali primitivi. Nostra esperienza. Minerva Chir 1994; 49: 43-46.

2. AIZCORBE M, ARMENDÁRIZ P, COBO F y cols.: Presentación y comentario de cinco liposarcomas retroperitoneales. Cir Esp 1989; 5: 732-735.

3. GUZMÁN MARTÍNEZ-VALL PL, FERRERO DORIA R, LÓPEZ ALBA J y cols.: Liposarcoma retroperitoneal. A propósito de tres casos. Arch Esp de Urol 1997; 50 (5): 529-531.

4. VERA DONOSO CD, JIMÉNEZ CRUZ JF.: Tumores retroperitoneales primarios. En: Tratado de Urología. J.R. Prous Editores 1993; 71: 1323-1336.

5. DÍAZ DEL RIO M, ABASCAL J, HERRERA N y cols.: Tumores retroperitoneales primitivos: experiencia sobre 115 casos. Cir Esp 1991, 49: 129-136.

6. ASPIAZU ARNÁIZ P, MURO BIDAURRE I, DE FRUTOS GAMERO A y cols.: Tumores retroperitoneales. Liposarcoma mixoide retroperitoneal. Presentación de un nuevo caso. Arch Esp de Urol 2000; 53 (2): 170-173.

7. NAKASHIMA J, UENO M, NAKAMURA K et al.: Differential diagnosis of primary benign and malignant retroperitoneal tumors. Int J Urol 1997 sep; 4 (5): 441-446.

8. EILBER FC, EILBER KS, EILBER FR.: Retroperitoneal sarcomas. Curr Treat Options Oncol 2000 aug; 1 (3): 274-278.

Dr. R. Ferrero Doria

Avda. Albaida, 2 - Esc. 1, pta. 18

46870 Onteniente (Valencia)

(Trabajo recibido el 19 mayo de 2003) 